\section{Chromosome Size Differences May Affect Meiosis and Genome Size}

\author{
John Wang, ${ }^{1 *}$ Pei-Jiun Chen, ${ }^{2} \dagger$ George ]. Wang, ${ }^{3}$ Laurent Keller $^{1 *}$
}

$\mathrm{T}$ by deviations from the equal representation of parental alleles in the progeny (1). Weak transmission bias and violations of the independent assortment of chromosomes are harder to detect, however, because large broods and a careful exam-

$\mathrm{X}$ chromosome and of the shorter wt chromosome with the X. We refer to this phenomenon as skew.

To exclude the possibility that skew for $m I s 10$ was specific to this genetic construct or chromosome $\mathrm{V}$, we investigated segregation patterns for large insertion transgenes on the other four autosomes. All Transmission distortion has been identified

Skew may affect genome evolution and genome size. Given that hermaphrodites tend to inherit the shorter chromosome and because new populations of $C$. elegans are frequently initiated by hermaphrodites (3), genome size reduction may occur over evolutionary time. Simulations of hermaphroditic mating, hermaphroditic selfingonly, and gonochoristic mating systems (2) (fig. S3) showed that skew led to a significant reduction in genome size in the hermaphroditic mating system $\left(P=2.5 \times 10^{-13}\right.$, one-sample $t$ test $)$ but not in the gonochoristic or hermaphroditic selfing-only systems (both $P>0.27$, one-sample $t$ test).

These observations of insertion disjoining from the $\mathrm{X}$, causing genome size reduction in male/hermaphrodite relative to gonochoristic mating systems, is consistent with genome sizes in the genus Caenorhabditis. Hermaphroditism within the genus has evolved independently at least twice (4). All three male/female species tested have genome sizes of $>130 \mathrm{Mb}$, whereas both sequenced hermaphroditic species have genome sizes of $\sim 100 \mathrm{Mb}(5-7)$. Thus, in at least two cases, the evolution of hermaphroditism was associated with a convergent reduction in genome size relative to the ancestral male/female species.

\section{References and Notes}

1. T. W. Lyttle, Annu. Rev. Genet. 25, 511 (1991).

2. Materials and methods are available as supporting material on Science Online.

3. A. Barrière, M. A. Félix, Curr. Biol. 15 1176 (2005)

4. K. Kiontke et al., Proc. Natl. Acad. Sci. U.S.A. 101, 9003 (2004).

5. E. S. Haag et al., Trends Genet. 23, 101 (2007).

6. L. D. Stein et al., PLoS Biol. 1, E45 (2003).

7. The C. elegans Sequencing Consortium, Science 282, 2012 (1998).

8. We thank W. Herr, P. Gönczy, A. W. Murray, and A. M. Villeneuve for comments on the manuscript; alternate chromosomes by sex. $N$, number of males tested; $n$, total individuals scored.

ination of the transmission ratios are necessary. We investigated whether weak violations of chromosome transmission or assortment exist in the nematode Caenorhabditis elegans, which is characterized by an XX/XO sex determination system.

We crossed males heterozygous for $m I s 10$ [an integrated transgene on chromosome V expressing green fluorescent protein (GFP)] to wild-type hermaphrodites and found sex-biased inheritance of $m I s 10$ (Fig. 1A, $P=9.8 \times 10^{-189}, \chi^{2}$ test). Sons were more likely to inherit the $m I s 10$ allele, whereas hermaphrodite daughters were more likely to receive the wild-type $(w t)$ allele. This was not due to the preferential transmission of one particular chromosomal allele, because the number of total offspring inheriting $\mathrm{mIS} 10$ was not different from that inheriting wt $(P=0.39$, binomial test). We also ruled out mortality of males bearing the $w t$ allele and of hermaphrodites bearing the $m I s 10$ allele, because we observed no dead larvae and only a few dead eggs. Thus, there is preferential segregation of the longer $m I s 10$-bearing chromosome away from the four insertion transgenes also preferentially segregated away from the $\mathrm{X}$ chromosome (Fig. 1B) with significantly differing magnitudes of skew among the insertion lines $\left(P<2.2 \times 10^{-16}, \chi^{2}\right.$ test $)$ and with transmission bias ratios ranging from $1.42(\mathrm{mls} / 3)$ to 6.55 ( $\mathrm{mIs} 10)$. Skew also occurred in males heterozygous for large deletions (Fig. 1C, $n D f 24 I$ and $t D f I I V$ ).

Because these insertions and deletions (indels) are relatively large (range $\sim 615 \mathrm{~kb}$ to $\sim 7.3 \mathrm{Mb}$, table $\mathrm{S} 1$ ), we tested whether smaller indels also exhibited skew by using a small insertion (ruIs 38, 33 kb) and three single-gene deletions (unc-30, unc-47, and unc-63, range from $\sim 1$ to $\sim 1.5 \mathrm{~kb}$ ). In the four cases, the shorter chromosome also segregated with the $\mathrm{X}$ chromosome, the skew being significant for $r u I s 38$, unc-47, and unc-63 (fig. S1). The magnitude of bias for these four indels (1.02 to 1.21) was smaller than for the larger indels. We also observed no difference regarding the parent of origin of an indel on skew (2). Overall, there was a significant positive correlation between indel size and transmission bias ratio (fig. S2, $P=0.006$, Spearman's rank correlation test). S. K. Kim and R. E. Ellis for discussions; W. Herr, in whose laboratory the experiments were performed; the Caenorhabditis Genetics Center, funded by the NIH National Center for Research Resources, for strains; the University of Lausanne Cellular Imaging Facility; and the Vital-IT Center for high-performance computing. Supported by grants from the Swiss National Science Foundation to L.K. and W. Herr. L.K. thanks S. Leibler for hosting him during his sabbatical at Rockefeller University.

\section{Supporting Online Material}

www.sciencemag.org/cgi/content/full/329/5989/293/DC1

Materials and Methods

SOM Text

Figs. S1 to S3

Table S1

References

26 March 2010; accepted 24 May 2010

10.1126/science.1190130

${ }^{1}$ Department of Ecology and Evolution, University of Lausanne, CH-1015 Lausanne, Switzerland. ${ }^{2}$ Center for Integrative Genomics, University of Lausanne, CH-1015 Lausanne, Switzerland. ${ }^{3}$ Howard Hughes Medical Institute and Department of Biology, Stanford University, Stanford, CA 94305-5020, USA.

*To whom correspondence should be addressed. E-mail: John.Wang@unil.ch (J.W.); Laurent.Keller@unil.ch (L.K.) †Present address: TopoGenomics Incorporated, Taipei, Taiwan. 